\section{The relationship between the cornea and the optic disc}

\begin{abstract}
Objective To evaluate the structural relationship between the cornea and the optic disc in normal subjects.

Methods This hospital-based observational study included 205 eyes from 205 individuals who were diagnosed as normal at our glaucoma clinic. The subjects underwent an eye examination, optic disc imaging with optic disc photography, optical coherence tomography, IOL master, specular microscopy, and ultrasound corneal pachymetry.

Results In univariate regression models (Pearson's correlation coefficient), the cup-to-disc (CD) ratio showed a negative correlation with corneal curvature $(r=-0.315, P<0.001)$ and central corneal thickness $(r=-0.206, P=0.005)$, and a positive correlation with white-to-white diameter (horizontal limbus to limbus distance, $r=0.215, P=0.003)$. In multiple linear regression models with $C D$ ratio as the dependant parameter, the CD ratio was still significantly associated with corneal curvature $(\beta=-0.205$, $P=0.011)$ and white-to-white diameter $(\beta=0.207, P=0.010)$. The central corneal thickness failed to show statistical significance, but did show a negative correlation with borderline significance $(\beta=-0.133, P=0.075)$. Conclusions Eyes with a large CD ratio have large and flat corneas; this may suggest that there is a structural relationship between the cornea and the optic disc. These results can be helpful in analysing the anatomical relationship between the cornea and the optic disc.
\end{abstract}

Eye (2010) 24, 1653-1657; doi:10.1038/eye.2010.98; published online 23 July 2010

Keywords: cornea; cup to disc ratio; optic disc; white to white diameter

\section{Introduction}

The cornea is the structure corresponding to the anterior opening of the eyeball and it is
JM Kim ${ }^{1}, \mathrm{KH}$ Park ${ }^{2}, \mathrm{SH} \mathrm{Kim}{ }^{2}, \mathrm{JH}_{\text {Kang }}{ }^{3}$

and SW Cho'

surrounded by the sclera. The eyeball gains its structure through the continuity of the cornea, sclera, and optic nerve lamina. The glial part of the optic nerve lamina cribrosa is embryologically neuroectoderm, but mesenchyme originates from the neural crest. Because the corneal stroma, corneal endothelium, optic nerve lamina cribrosa, and meningeal sheath all differentiate from the neural crest, they may be embryologically related to each other. ${ }^{1}$

Several studies have suggested the possibility of a relationship between the optic nerve and the cornea. One study suggested that an abnormal-shaped optic nerve is strongly related to corneal astigmatism. ${ }^{2}$ Other researchers have reported that a tilted disc may be caused by the closure of an imperfect embryonic fissure and that corneal astigmatism may result from the influence of genetic factors on the development of the cornea in tilted disc syndrome patients. ${ }^{3-6}$ There have been many reports on the relationship between the central corneal thickness and the optic nerve and glaucoma. Some studies have reported that thin corneas may have an association with weak optic nerve heads and that this may be related to a thin lamina cribrosa. ${ }^{7,8}$ Furthermore, some studies have shown that the development and progression of glaucoma correlates with central corneal thickness; ${ }^{9}$ other studies refute this claim. ${ }^{10,11}$ Wells et $a l^{11}$ insisted that corneal hysteresis, but not corneal thickness, correlates with the vulnerability of the optic nerve surface.

Researchers generally agree that the lamina cribrosa is important in glaucoma. ${ }^{12-15}$

Nevertheless, in vivo clinical clues regarding the correlated parameters of the lamina cribrosa are limited. ${ }^{13,16}$ We hypothesised that the presence of lamina cribrosa in optic nerve roots outside the eye may correlate with the optic disc area. The aim of this study is to determine whether there is a structural relationship between the cornea and the optic nerve.
${ }^{1}$ Department of Ophthalmology, Sungkyunkwan University School of Medicine, Kangbuk Samsung Hospital, Seoul, Korea

2Department of Ophthalmology, Seoul National University College of Medicine, Seoul National University Hospital, Seoul, Korea

${ }^{3}$ Department of Ophthalmology, Kyung Hee University College of Medicine, East-West Neo Medical Center, Seoul, Korea

Correspondence: KH Park, Department of Ophthalmology, Seoul National University College of Medicine, Seoul National University Hospital, 28 Yongon-dong, Chongno-gu, Seoul 110-744, Korea. Tel: + 82-2-2072-2438; Fax: + 82-2-741-3187. E-mail: kihopark@ snu.ac.kr

Received: 18 September 2009

Accepted in revised form: 28 May 2010 Published online: 23 July 2010 


\section{Patients and methods}

Our study included 205 normal eyes from 205 subjects who visited the Kangbuk Samsung hospital for routine checkup, spectacle correction, or glaucoma work-up between January 2007 and October 2007, and who had no ocular pathology. Among the subjects, 95 were female and 110 were male. This study was approved by the Institutional Review Board of the Kangbuk Samsung hospital and was performed in accordance with the tenets of the Declaration of Helsinki.

Uncorrected visual acuity was measured with Snellen charts. Automatic refractometry (RK F-1; Cannon, Tochigiken, Japan) was performed. Intraocular pressure (IOP) was measured with a Goldman applanation tonometer (Haag-Streit AT 900, Haag-Streit AG, Koeniz, Switzerland) by an experienced ophthalmologist using the average of the three measurements. A slit-lamp examination was performed by one glaucoma specialist. An IOL master (Carl Zeiss Meditec, Dublin, CA, USA) of the globe was performed. The measurements of white-towhite diameter (horizontal corneal limbus to limbus distance), corneal curvature, and axial length were taken using the IOL master without pupil dilation, and the horizontal white-to-white diameter was used as the corneal white-to-white diameter. Central corneal thickness (CCT) was measured using an ultrasonic pachymeter (Cinescan; Quantel medical, Bozeman, Montana, USA) and the average of three measurements. Corneal endothelial cell density was measured using a specular biomicroscope (Noncon Robo; Konan Medical, Inc., Hyogo, Japan) and the average of the three measurements. Digital red-free fundus photographs of the retina and stereo disc photographs were taken using a nonmydriatic fundus camera (Visucam; Carl Zeiss Meditec) to exclude glaucoma or other retinal diseases. In addition, optic disc photographs were examined to determine the cup-to-disc (CD) ratio. One glaucoma specialist calculated the CD ratio using the Visupac programme (version 4.2; Carl Zeiss Meditec), which can measure the cup and disc area (Figure 1). We measured the optic disc structures by outlining the borders of the optic disc and the optic cup on the computer screen and then confirmed the margin of the optic disc and cup, with stereoscopic findings using a stereoscopic viewer. As there was no adjustable function of axial length or refractive error, it was impossible to estimate the real area. As such, the CD ratio was used for analysis. The CD ratio was calculated (cup area/disc area). We measured the retinal nerve fibre layer (RNFL) thickness using the Stratus OCT (Carl Zeiss Meditec).

Inclusion criteria included normal subjects with clear disc photography, without any history of pathology affecting the disc morphology in either eye. Normal eyes

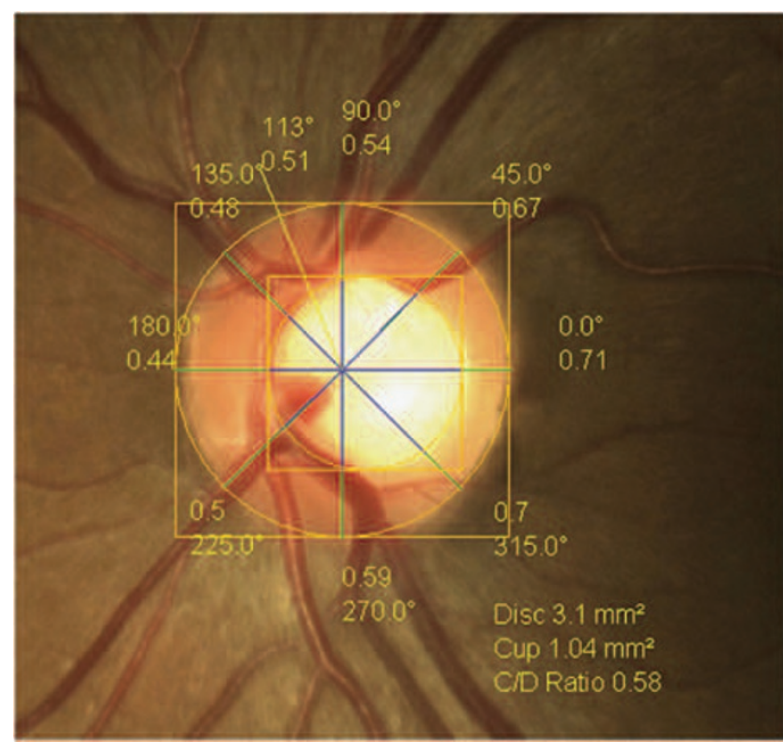

Figure 1 Cup and disc ratio measurement.

were defined as those eyes with normal visual acuity (at least 20/30 corrected visual acuity), normal IOP (below $20 \mathrm{~mm} \mathrm{Hg}$ ), and without disc abnormalities on disc photography or RNFL defects on red-free photography. If the $\mathrm{CD}$ ratio was large $(>0.5)$, we confirmed their status with OCT and visual field testing.

Exclusion criteria included a history of intraocular disease, ocular injuries, intraocular or extraocular operation, optic nerve disease, or abnormal optic nerve shape (eg coloboma, optic hypoplasia, morning glory disc anomaly). Eyes with IOPs $>19 \mathrm{~mm} \mathrm{Hg}$ and 20/30 or worse visual acuity were also excluded. Ambiguous disc cases, which had an unclear boundary of the optic disc, cup, or rim were excluded. Patients with a tilted disc or glaucoma were also excluded.

We analysed the data of the right eye to eliminate selection bias. Pearson's correlation analysis and multiple regression analysis were used. Statistical analysis was performed using a commercially available statistical software package (SPSS version 15.0; SPSS, Chicago, IL, USA). Confidence intervals were generated. All $P$-values were two-sided and were considered statistically significant when the value was $<0.05$.

\section{Results}

The mean patient age was $49.2 \pm 14.9$ years (range: $18-82$ ). The CD ratio was $0.56 \pm 0.12$ (range: $0.30-0.85$ ). The mean CCT was $535.8 \pm 36 \mu \mathrm{m}$ (range: $410-620$ ), the mean axial length was $24.34 \pm 1.48 \mathrm{~mm}$ (range: 21.18-29.41), and the mean corneal white-to-white diameter was $11.96 \pm 0.56 \mathrm{~mm}$ (range: 10.60-12.90; Table 1).

The CD ratio correlated well with the disc area, which was measured using the Visupac programme 
Table 1 Patient demograhics

\begin{tabular}{lc}
\hline & Mean $\pm S D$ (range) \\
\hline Age (years) & $49.35 \pm 14.86(18-82)$ \\
Cup-to-disc ratio & $0.56 \pm 0.12(0.3-0.85)$ \\
Central corneal thickness $(\mu \mathrm{m})$ & $535.7 \pm 36(420-620)$ \\
Axial length $(\mathrm{mm})$ & $24.34 \pm 1.48(21.18-29.41)$ \\
Corneal white-to-white & $11.90 \pm 0.49(10.60-12.80)$ \\
diameter (mm) & \\
Corneal curvature (diopters) & $43.71 \pm 1.56(16.50-48.29)$ \\
\hline
\end{tabular}

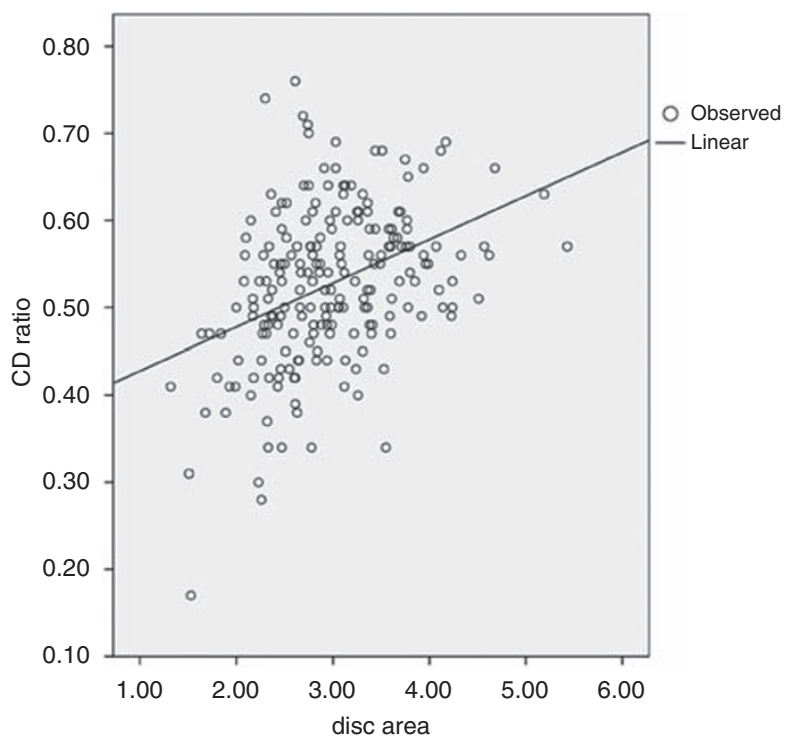

Figure 2 Scattergram showing the correlation between disc area measured by visupac programme and cup-to-disc ratio.

$(P<0.001$; Figure 2). In univariate regression models (Table 2), CD ratio showed a negative correlation with corneal curvature $(r=-0.315, P<0.001$; Figure 3$)$ and CCT $(r=-0.206, P=0.005)$, and a positive correlation with white-to-white diameter $(r=0.215, P=0.003$; Figure 4). The axial length correlated with the refractive error $(r=-0.811, P<0.001)$, corneal curvature $(r=-0.456, P<0.001)$, and white-to-white diameter $(r=0.251, P=0.001)$. The white-to-white diameter correlated with the $\mathrm{CD}$ ratio $(r=0.215, P=0.003)$, corneal curvature $(r=-0.392, P<0.001)$, axial length $(r=0.251, P=0.001)$, and CCT $(r=-0.148, P=0.042)$. The corneal endothelium (number: $2698 \pm 316$; mean area: $142.7 \pm 56.4)$ did not correlate with the CD ratio or white-to-white diameter. In multiple linear regression models (Table 3) with the CD ratio as the dependant parameter, the $\mathrm{CD}$ ratio was still significantly associated with corneal curvature $(\beta=-0.205, P=0.011)$ and white-to-white diameter $(\beta=0.207, P=0.010)$. The CCT failed to show statistical significance $(\beta=-0.133$, $P=0.075)$. Other factors did not show a significant
Table 2 Associations between CDR, CCT, ECC, AXL, Curv, and WWD (univariate analysis, Pearson's correlation)

\begin{tabular}{llrrrrr}
\hline & & $C D R^{\mathrm{a}}$ & $C C T^{\mathrm{b}}$ & $A X L^{\mathrm{c}}$ & Curv $^{\mathrm{d}}$ & $W^{\mathrm{e}}$ \\
\hline $\mathrm{CDR}^{\mathrm{a}}$ & Correlation & & -0.200 & 0.095 & -0.323 & 0.199 \\
& Significance & & 0.075 & 0.208 & 0 & 0.008 \\
$\mathrm{CCT}^{\mathrm{b}}$ & Correlation & -0.200 & & -0.047 & 0.153 & -0.111 \\
& Significance & 0.075 & & 0.527 & 0.046 & 0.141 \\
$\mathrm{AXL}^{\mathrm{c}}$ & Correlation & 0.095 & -0.047 & & -0.460 & 0.227 \\
& Significance & 0.208 & 0.527 & & 0 & 0.003 \\
$\mathrm{Curv}^{\mathrm{d}}$ & Correlation & -0.323 & 0.153 & -0.460 & & -0.405 \\
& Significance & 0 & 0.046 & 0 & & 0 \\
$\mathrm{WWD}^{\mathrm{e}}$ & Correlation & 0.199 & -0.111 & 0.227 & -0.405 & \\
& Significance & 0.008 & 0.141 & 0.003 & 0 & \\
\hline
\end{tabular}

${ }^{\mathrm{a}} \mathrm{Cup}$-to-disc ratio.

${ }^{\mathrm{b}}$ Central corneal thickness.

'Axial length.

${ }^{\mathrm{d} C}$ Corneal curvature.

${ }^{\mathrm{e}}$ White-to-white diameter.

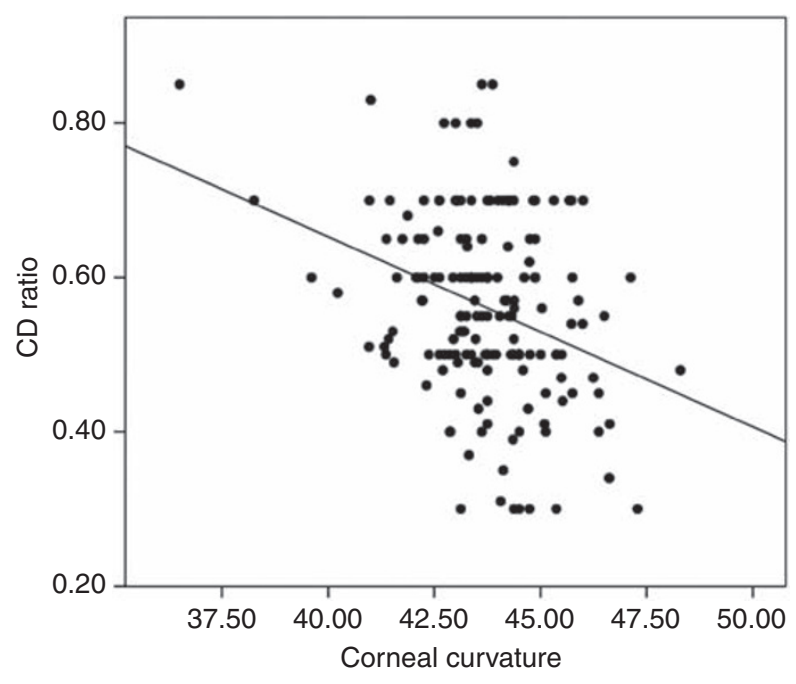

Figure 3 Scattergram showing the correlation between corneal curvature and cup-to-disc ratio.

correlation with the $\mathrm{CD}$ ratio, including the RNFL thickness measurements made using Stratus OCT.

\section{Discussion}

Our results show that eyes with large $C D$ ratios have large and flat corneas. A large $\mathrm{CD}$ ratio in normal subjects is usually associated with a large disc. A large disc corresponds to a large posterior opening, and a large white-to-white diameter corresponds to a large anterior opening. We also considered that large discs may be related to large lamina cribrosas and that large lamina cribrosas may be preferentially thin. This assumption is based on our hypothesis that ocular tissue does not grow, but rather expands, after the infantile period. ${ }^{17}$ 


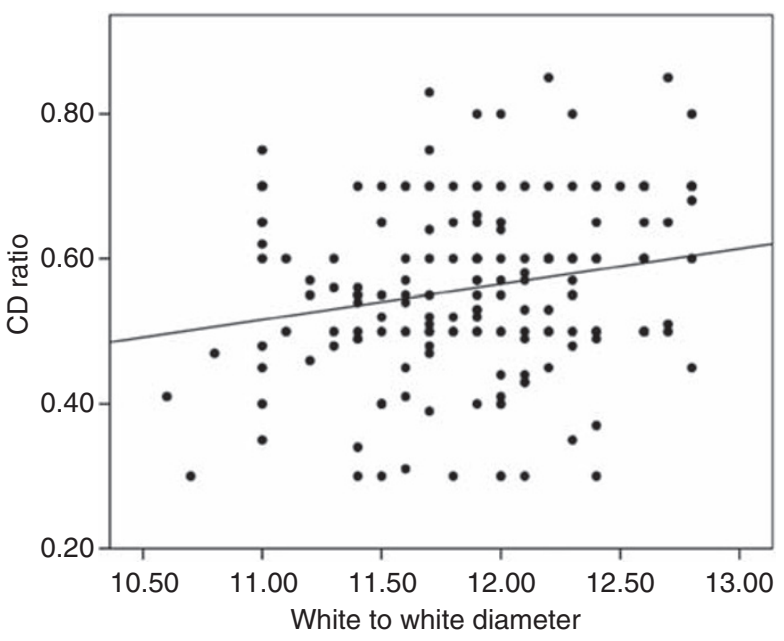

Figure 4 Scattergram showing the correlation between white-to-white diameter and cup-to-disc ratio.

Table 3 Results of the multiple linear regression analysis with cup-to-disc ratio

\begin{tabular}{lcc}
\hline & Coefficients & Sig. $^{\text {a }}$ \\
\hline CCT $^{\mathrm{b}}$ & -0.133 & 0.083 \\
WWD $^{\mathrm{c}}$ & 0.207 & 0.010 \\
Corneal curvature $^{\mathrm{d}}$ & -0.133 & 0.075 \\
\hline
\end{tabular}

${ }^{\text {aStatistical significance. }}$

${ }^{\mathrm{b}}$ Central corneal thickness.

'White-to-white diameter.

${ }^{\mathrm{d}}$ Corneal curvature.

Glaucoma is a progressive optic neuropathy and, therefore, many clinicians have an interest in the status of the optic nerve. Many reports have suggested that the cornea is related to the optic nerve in some way; some clinicians have looked for a relationship between glaucoma and CCT. ${ }^{7-10,18}$ A recent study found that CCT is not related to the risk of developing a glaucomatous visual field loss or progression, ${ }^{19}$ and a histomorphometric study failed to find an association between CCT and the thickness of the lamina cribrosa. ${ }^{12,20}$ However, these results did not provide a definitive answer regarding the relationship between the cornea and the optic disc. According to Pakravan et $a l^{7}$ the size of the optic nerve is large and deformation of the optic nerve is more frequent when the central cornea is thin. Cankaya et $a l^{8}$ also showed that CCT has a structural relationship with the optic nerve head in healthy eyes. Our results unambiguously show that the cornea is related to the optic disc. The corneal endothelium and CCT were not related to the axial length or to the optic disc size; however, corneal curvature and white-to-white diameter were related to the disc size. These results indicate that subjects with large discs will likely have large white-to-white diameters and flat corneas. As the eyeball grows, the anterior scleral opening becomes larger. We can think of the cornea as flattening, but not growing.

In myopic eyes, if the eyeball grows and the axial length elongates, the white-to-white diameter (anterior scleral opening) increases and the cornea flattens. ${ }^{17}$ Moreover, the posterior opening and the optic disc are larger, and the lamina cribrosa becomes larger and thinner. The secondary thinning of the lamina cribrosa occurs and may lead to an increased risk of glaucoma. ${ }^{15}$ Wells et $a l^{11}$ showed the relationship between the cornea and the optic disc in vivo using Heidelberg Retina Tomography II (Heidelberg Engineering, GmbH, Dossenheim, Germany). According to their results, corneal hysteresis, but not CCT or other anterior segment parameters, was associated with increased deformation of the optic nerve surface during transient elevations of IOP. In the present study, corneal thickness and corneal endothelium were not affected by axial length or disc size. We think that this may be responsible for the difference in the timing of growth between the anterior segment and posterior segment of the globe. The cornea remains unchanged after 2 years in normal eyes. ${ }^{21}$ As axial length increases, passive widening (not stretching) of the anterior scleral opening (white-to-white diameter) will cause flattening of the cornea without stretching or thinning. In the present study, the white-to-white diameter showed significant correlations with CD ratio, corneal curvature, and axial length. These results suggest that the optic disc growth is in proportion to the white-to-white diameter and that subjects with large discs will likely have large white-to-white diameters and flat corneas. However, we failed to show a statistically significant relationship between increased axial length and a large CD ratio $(r=0.101, P=0.180)$. This result differs from another report which found that increased disc area is associated with increased axial length. ${ }^{22}$ These different results may be because of racial differences (all our subjects were Korean), relatively small sample size, or the use of different measuring devices. Also, the exclusion of subjects with tilted optic discs may have influenced our results. This difference could also be explained by the variable patterns of eye growth associated with myopia, as a long eye may have a small disc if its width and height do not increase as much as its length. ${ }^{22-24}$ A large disc that does not have a long axial length is the result of more horizontal elongation than axial elongation. Another possibility is that the optic disc may not be the primary location of stretching as the eyeball grows. The large degree of peripapillary atrophy in high myopic eyes could be one example. However, this hypothesis needs further investigation. An anatomical relationship between a large cornea, large optic disc, long axial length, and thin lamina cribrosa is anticipated and 
may be associated with the risk of development or progression of glaucoma.

Limitations of this study include that the optic disc size was obtained by indirect calculation using the $\mathrm{CD}$ ratio instead of measuring the real disc size. However, to reduce errors, we estimated the $\mathrm{CD}$ ratio using the area of the cup and disc, which were measured using the Visupac programme. It is difficult to accurately measure the disc size; using the CD ratio is helpful in obtaining accurate values. Studies using the real optic disc area may be required for more accurate results in glaucoma patients. Techniques to directly measure the real optic disc size, the real size of the lamina cribrosa, and the thickness of the lamina cribrosa may be useful.

We attempted to identify a parameter related to the lamina cribrosa, because the lamina cribrosa is the most important anatomical region in the development and progression of glaucoma. The present study suggests that a larger white-to-white diameter is related to a larger CD ratio. To the best of our knowledge, this study represents an initial attempt to elucidate a probable relationship between corneal size (white-to-white diameter) and optic disc size. We suggest that the white-to-white diameter can be used in evaluating the lamina cribrosa; this finding may be of importance in determining the pathogenesis of glaucomatous optic neuropathy.

\section{Summary}

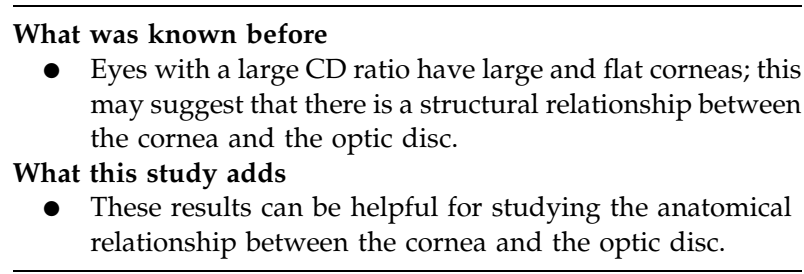

\section{Conflict of interest}

The authors declare no conflict of interest.

\section{References}

1 Sellheyer K, Spitznas M. Development of the human sclera. A morphological study. Graefes Arch Clin Exp Ophthalmol 1988; 226: 89-100.

2 Jonas JB, Kling F, Gründler AE. Optic disc shape, corneal astigmatism, and amblyopia. Ophthalmology 1997; 104: 1934-1937.

3 Bozkurt B, Irkec M, Gedik S, Orhan M, Erdener U. Topographical analysis of corneal astigmatism in patients with tilted-disc syndrome. Cornea 2002; 21: 458-462.

4 Apple DJ. New aspects of colobomas and optic nerve anomalies. Int Ophthalmol Clin 1984; 24: 109-121.

5 Lempert P, Porter L. Dysversion of the optic disc and axial length measurements in a presumed amblyopic population. J AAPOS 1998; 2: 207-213.
6 Young SE, Walsh FB, Knox DL. The tilted disk syndrome. Am J Ophthalmol 1976; 82: 16-23.

7 Pakravan M, Parsa A, Sanagou M, Parsa CF. Central corneal thickness and correlation to optic disc size: a potential link for susceptibility to glaucoma. Br J Ophthalmol 2007; 91: 26-28.

8 Cankaya AB, Elgin U, Batman A, Acaroglu G. Relationship between central corneal thickness and parameters of optic nerve head topography in healthy subjects. Eur J Ophthalmol 2008; 18: 32-38.

9 Herndon LW, Weizer JS, Stinnett SS. Central corneal thickness as a risk factor for advanced glaucoma damage. Arch Ophthalmol 2004; 122: 17-21.

10 Leske MC, Heijl A, Hussein M, Bengtsson B, Hyman L, Komaroff E, Early Manifest Glaucoma Trial Group. Factors for glaucoma progression and the effect of treatment: the early manifest glaucoma trial. Arch Ophthalmol 2003; 121: 48-56.

11 Wells AP, Garway-Heath DF, Poostchi A, Wong T, Chan KC, Sachdev $\mathrm{N}$ et al. Corneal hysteresis but not corneal thickness correlates with optic nerve surface compliance in glaucoma patients. Invest Ophthalmol Vis Sci 2008; 49: 3380-3386.

12 Jonas JB, Martus P, Budde WM, Jünemann A, Hayler J. Small neuroretinal rim and large parapapillary atrophy as predictive factors for progression of glaucomatous optic neuropathy. Ophthalmology 2002; 109: 1561-1567.

13 Jonas JB, Holbach L. Central corneal thickness and thickness of the lamina cribrosa in human eyes. Invest Ophthalmol Vis Sci 2005; 46: 1275-1279.

14 Jonas JB, Berenshtein E, Holbach L. Anatomic relationship between lamina cribrosa, intraocular space, and cerebrospinal fluid space. Invest Ophthalmol Vis Sci 2003; 44: 5189-5195.

15 Jonas JB, Berenshtein E, Holbach L. Lamina cribrosa thickness and spatial relationships between intraocular space and cerebrospinal fluid space in highly myopic eyes. Invest Ophthalmol Vis Sci 2004; 45: 2660-2665.

16 Jonas JB, Gusek GC, Guggenmoos-Holzmann I, Naumann GO. Size of the optic nerve scleral canal and comparison with intravital determination of optic disc dimensions. Graefes Arch Clin Exp Ophthalmol 1988; 226: 213-215.

17 Parsa CF. Congenital optic disc anomalies. In: Albert DM, Miller JW (eds). Albert and Jacobiec's Principles and Practice of Ophthalmology, 3rd edn. Saunders/Elsevier, Philadelphia, pp 2008; 4271-4275.

18 Choi HJ, Kim DM, Hwang SS. Relationship between central corneal thickness and localized retinal nerve fiber layer defect in normal-tension glaucoma. J Glaucoma 2006; 15: 120-123.

19 Araie M, Sekine M, Suzuki Y, Koseki N. Factors contributing to the progression of visual field damage in eyes with normaltension glaucoma. Ophthalmology 1994; 101: 1440-1444.

20 Jonas JB, Hayreh SS, Tao Y. Central corneal thickness and thickness of the lamina cribrosa and peripapillary sclera in monkeys. Arch Ophthalmol 2009; 127: 1395-1396.

21 Larsen JS. The sagittal growth of the eye. 3. Ultrasonic measurement of the posterior segment (axial length of the vitreous) from birth to puberty. Acta Opthalmol 1971; 49: 873-886.

22 Atchison DA, Jones CE, Schmid KL, Pritchard N, Pope JM, Strugnell WE et al. Eye shape in emmetropia and myopia. Invest Ophthalmol Vis Sci 2004; 45: 3380-3386.

23 Oliveira C, Harizman N, Girkin CA, Tello C, Liebmann JM, Ritch R. Axial length and optic disc size in normal eyes. Br J Ophthalmol 2007; 91: 37-39.

24 Park SH, Park KH, Kim JM, Choi CY. The relation between axial length and ocular parameters. Ophthalmologica 2010; 224: 188-193. 